Math. Model. Nat. Phenom.

Vol. 6, No. 1, 2011, pp. 163-187

DOI: $10.1051 / \mathrm{mmnp} / 20116109$

\title{
Modeling Dryland Landscapes
}

\author{
E. Meron ${ }^{1}$ \\ Institute for Dryland Environmental Research, BIDR, Ben-Gurion University, \\ Sede Boqer Campus, 84990, Israel \\ and \\ Department of Physics, Ben-Gurion University, Beer-Sheva 84105, Israel
}

\begin{abstract}
The discovery of nearly periodic vegetation patterns in arid and semi-arid regions motivated numerous model studies in the past decade. Most studies have focused on vegetation pattern formation, and on the response of vegetation patterns to gradients of the limiting water resource. The reciprocal question, what resource modifications are induced by vegetation pattern formation, which is essential to the understanding of dryland landscapes, has hardly been addressed. This paper is a synthetic review of model studies that address this question and the consequent implications for inter-specific plant interactions and species diversity. It focuses both on patch and landscape scales, highlighting bottom-up processes, where plant interactions at the patch scale give rise to spatial patterns at the landscape scale, and top-down processes, where pattern transitions at the landscape scale affect inter-specific interactions at the patch scale.
\end{abstract}

Key words: vegetation patchiness, pattern formation, ecosystem engineer, woody-herbaceous systems, competition, facilitation, mathematical modeling.

AMS subject classification: $97 \mathrm{M} 10$

\section{Introduction}

Landscapes in arid and semi-arid regions are mosaics of vegetation patches that differ in biomass production, resource concentration, and species richness [1]. Understanding the dynamics of such landscapes under various environmental conditions is highly significant for assessing the likelihood

\footnotetext{
${ }^{1}$ E-mail: ehud@bgu.ac.il
} 
of desertification [2, 3, 4] and biodiversity-loss phenomena [5], for studying preventive measures, and for devising restoration practices for degraded areas $[6,7]$.

Research efforts in this direction have involved field and laboratory experiments $[8,9,10]$ as well as mathematical modeling and model studies [11]. Field and laboratory experiments are often limited by their duration; the longer experiments endure a few tens of years, whereas patch dynamics in drylands may proceed on much longer time scales. Field experiments are also inherently limited by the uncontrollable environments in which they are carried out, while controlled laboratory experiments are limited by their spatial extent. Mathematical models circumvent these limitations. They allow identifying asymptotic behaviors, studying ecological processes at landscape scales, and elucidating mechanisms by controlling biotic and abiotic factors. In addition they allow studying various scenarios of ecosystem dynamics, and testing management practices. Mathematical modeling, however, has its own limitations. Most significant is the simplification, or quite often the oversimplification, of the complex reality that it entails.

Ecological processes generally proceed across different levels of organization and spatial scales. These important aspects of ecosystem complexity have to be taken into account in modeling the dynamics of dryland landscapes and applying the models to the questions of desertification and biodiversity-loss. Organizational hierarchy in dryland landscapes ranges from the organism level, i.e. the level of an individual plant, to the population level, a group of individuals belonging to the same species, to the community level, a group of populations of different species, and up to the ecosystem level that refers to a community of organisms interacting among themselves and with their non-living environment. Spatial variability in dryland landscapes begins at the patch scale with spatial biomass, resource and species distributions, and extends to the landscape scale, with various forms of patch configurations.

A characteristic feature of complex systems is the emergence of new properties that do not exist at the level of the system's constituents. A particularly significant property of this kind in the context of dryland landscapes is the possible emergence of symmetry breaking vegetation patterns in large populations or communities that extend over landscape scales. Various forms of vegetation patterns have been observed in arid and semi arid regions $[4,12,13]$. Many of the observed patterns have characteristic scales, suggesting that they are results of self organization phenomena, rather than the imprint of random environmental heterogeneities. The characteristic length can vary from $10 \mathrm{~cm}$ for grass patterns, as shown in Fig. 1, to $10 \mathrm{~m}$ and higher for woody vegetation.

Dryland vegetation consists primarily of woody vegetation (shrubs) and herbaceous vegetation (annuals and perennials). Quite often vegetation pattern formation is induced by the woody life form. This symmetry breaking process not only leads to biomass patterns; it also redistributes the limiting water resource, thereby affecting the herbaceous vegetation in terms of spatial distribution, community structure and diversity. Understanding the dynamics of dryland landscapes therefore calls for models that capture vegetation pattern formation by woody life forms, the associated redistribution of the water resource, and the consequent response of the herbaceous life form.

This paper is a synthetic review of model studies of dryland landscapes based on the model that has been introduced by Gilad et al. [14] and extensions thereof [15, 16, 17]. The review presents the model in a wider context than those considered in earlier studies (section 2.), and describe a few applications thereof, including vegetation pattern formation (section 3.), plants as ecosystem 
engineers (section 4.), and dynamics of woody-herbaceous systems (section 5.).

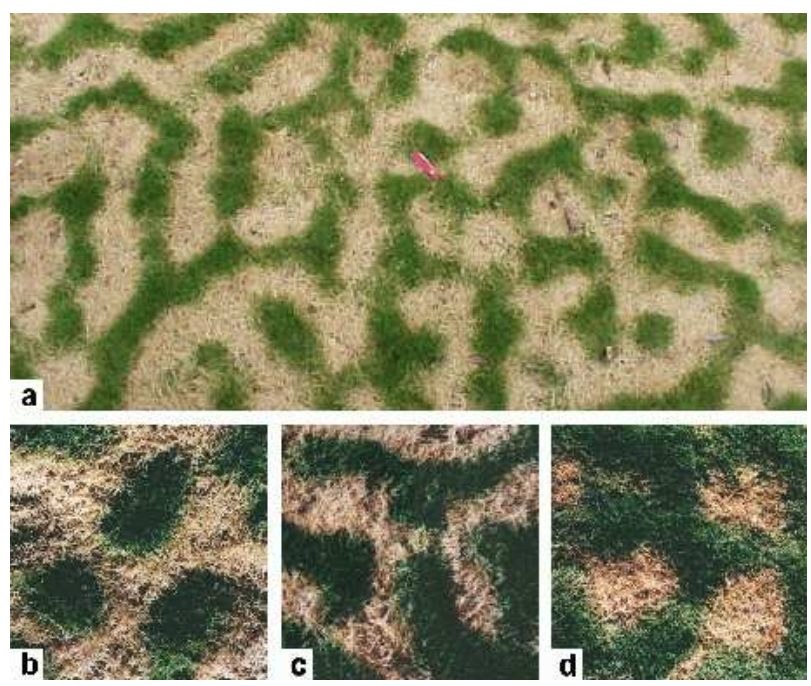

Figure 1: Patterns of Paspalum vaginatum observed in the Northern Negev ( $200 \mathrm{~mm}$ mean annual rainfall): a labyrinth-like pattern (a) and closeups showing spots (b), stripes (c) and gaps (d). The typical distance between spots and stripes is about $0.1 \mathrm{~m}$. From [3].

\section{The general model}

\subsection{The ecological and physical context}

We consider landscapes where vegetation growth is limited by water and possibly by additional non-consumable resources, such as light, or by energy-resource allocation to defense mechanisms [18]. We will mostly be concerned with two functional groups, representing woody and herbaceous vegetation. We assume that the landscape has a fixed topography. Thus, while past erosion-deposition processes, that shaped the topography, may have had significant effects on water flow and vegetation growth, the latter processes are assumed to have no significant feedback on the topography [19]. We define a "patch" to be an area covered by vegetation, which generally differs in its water content from the surrounding bare soil. By "bare" soil we mean areas that are not covered by vegetation. Such areas may still be covered by micro-organisms, most commonly cyanobacteria, that form biological crusts [20, 21]. Soil crusts generally reduce the infiltration rate of surface water into the soil, and thereby affect the spatial distribution of the limiting water resource [22]. They also affect the process of seed germination. These crust effects, however, will be parameterized as soil properties. Finally, while rainfall strongly affects vegetation growth, we will assume that the total vegetation biomass is too small to feed back on the atmosphere and affect the rainfall [23]. 


\subsection{The model equations}

The model contains $n+2$ dynamical variables: $n$ biomass variables, $B_{i}(\mathbf{X}, T)(i=1, \ldots, n)$, representing the above-ground biomass of $n$ life forms (in units of $\mathrm{kg} / \mathrm{m}^{2}$ ) as functions of the space and time coordinates, $\mathbf{X}=(X, Y)$ and $T$, a soil-water variable, $W(\mathbf{X}, T)$, describing the amount of soil water available to the plants per unit area of ground surface (in units of $\mathrm{kg} / \mathrm{m}^{2}$ ), and a surface water variable, $H(\mathbf{X}, T)$, describing the height of a thin water layer above ground level (in units of $\mathrm{mm}$ ). The life forms may represent species belonging to different functional groups, e.g. trees, shrubs, annuals etc., or species within a functional group, e.g. a community of annuals, or both types of species. We present here the model equations in non-dimensional form. A list of the dimensional parameters, their units and their meaning are given in Table 1. The definitions of the non-dimensional variables and parameters in terms of their dimensional counterparts are given in Table 2. We refer the reader to Gilad et al. [16] for more details. The model equations for the non-dimensional biomass variables, $b_{i}$, the soil-water variable, $w$, and the surface-water variable, $h$, are:

$$
\begin{aligned}
\frac{\partial b_{i}}{\partial t} & =\mathcal{G}_{b_{i}} b_{i}\left(1-b_{i}\right)-\mathcal{M}_{i} b_{i}+\mathcal{D}_{b_{i}} \quad i=1, \ldots, n \\
\frac{\partial w}{\partial t} & =\mathcal{I} h-\mathcal{L} w-\mathcal{G}_{w} \mathcal{F}+\delta_{w} \nabla^{2} w \\
\frac{\partial h}{\partial t} & =p-\mathcal{I} h-\nabla \cdot \mathcal{J}
\end{aligned}
$$

where $t$ is dimensionless time, $\mathbf{x}=(x, y)$ are dimensionless space coordinates, and $\nabla=\hat{\mathbf{x}} \partial / \partial x+$ $\hat{\mathbf{y}} \partial / \partial y$. We have used here calligraphic letters to distinguish quantities that depend on the dynamical variables from predetermined parameters.

In the equation for $b_{i}$, the quantity $\mathcal{G}_{b_{i}}\left[b_{i}, w\right]$ represents the growth rate of the $i$ th life form. It depends on the soil-water variable in a non-local manner as described in section 2.3.. The logistic form of the growth term represents limiting factors other than water, e.g. resource allocation to defense against pathogens. This factors are captured by the dimensional parameter $K_{i}$ that has been eliminated in rescaling the biomass $\left(b_{i}=B_{i} / K_{i}\right)$. The quantity $\mathcal{M}_{i}$ represents biomass decay rate due to mortality and consumer pressure, such as grazing. It is generally a function of the biomass variable. The quantity $\mathcal{D}_{b_{i}}\left[b_{i}\right]$ represents biomath growth due to seed dispersal or clonal growth. It is a non-local term that can be written as [24]

$$
\mathcal{D}_{b_{i}}(\mathbf{x}, t)=\int q\left(\mathbf{x}^{\prime}-\mathbf{x}\right) b_{i}\left(\mathbf{x}^{\prime}, t\right) d \mathbf{x}^{\prime}
$$

The kernel $q\left(\mathbf{x}^{\prime}-\mathbf{x}\right)$ determines the range of seed dispersal. When the range is short relative to the scale over which the biomass varies one may expand $b\left(\mathbf{x}^{\prime}, t\right)$ around $b(\mathbf{x}, t)$ in a Taylor series and keep terms to second order only. This results in the diffusive approximation, $\mathcal{D}_{b_{i}} \approx \delta_{b_{i}} \nabla^{2} b_{i}$, a rather crude approximation, that still captures the essential phenomenology of vegetation patchiness in the case of clonal growth, or in cases where long-distance seed-dispersal mechanisms [25], such as seed advection by winds, water flow and animals, are insignificant. 
In the equation for $w$ the quantity $\mathcal{I}\left(b_{i}\right)$ represents the infiltration rate of surface water into the soil, and the quantity $\mathcal{L}\left(b_{i}\right)$ represents soil-water loss due to evaporation. Both quantities depend on the biomass variables as described in section 2.3. The term $\mathcal{G}_{w}\left[b_{i}\right] \mathcal{F}(w)$ represents soil-water uptake (or transpiration) by all life forms. The uptake rate $\mathcal{G}_{w}\left[b_{i}\right]$ is a sum of nonlocal terms whose exact forms are discussed in section 2.3.. The function $\mathcal{F}(w)$ is generally a nonlinear increasing function of $w$ that approaches a constant value at high soil-water contents, e.g. Holling type-II or type-III functional dependence [26]. The last term in the soil-water equation describes soilwater diffusion in non-saturated soil. Implicit in this formulation is the assumption that the soil water content is fully available for uptake by the plants' roots, i.e. leakage losses associated with infiltration to deep soil layers are insignificant [17].

The equation for $h$ contains a source term $p$ representing precipitation, and a loss term, $-\mathcal{I} h$, representing infiltration of surface water into the soil. The flux $\mathcal{J}$ is given by

$$
\mathcal{J}=-2 \delta_{h} h \nabla(h+\zeta),
$$

and describes water overland flow over a ground surface characterized by a fixed topography function $\zeta$ (ground-level height). The dimensionless parameter $\delta_{h}$ represents a phenomenological coefficient inversely related to the friction between the surface water and the ground surface. Equations (2.3) and (2.5) were derived using shallow-water theory, assuming a Rayleigh friction term proportional to the flow velocity [14]. They form a particular case of the diffusive wave approximation of the shallow water equations

$$
\frac{\partial Z}{\partial t}-\nabla \cdot\left(\frac{(Z-\zeta)^{\alpha}}{\beta} \frac{\nabla Z}{|\nabla Z|^{1-\gamma}}\right)=\mathcal{P}
$$

where $Z=h+\zeta, \alpha=\gamma=1, \beta=\left(2 \delta_{h}\right)^{-1}$ and $\mathcal{P}=p-\mathcal{I} h$ [27]. For flat topographies Eqs. (2.3) and (2.5) reduce to a special case of the porous medium equation [28].

\subsection{Patch-scale feedbacks}

The model equations capture various feedbacks, between biomass and water and between aboveground and below-ground biomass, through the explicit forms of $\mathcal{G}_{b_{i}}, \mathcal{G}_{w}, \mathcal{I}$ and $\mathcal{L}$, as described below.

\subsubsection{Infiltration feedback}

This is a positive feedback between biomass and water due to increased infiltration rates of surface water into the soil in vegetation patches. The feedback is captured by several terms in the model equations: (a) the term $\mathcal{I} h$ in the equation for $w$, where the infiltration rate $\mathcal{I}$ is given by [16]

$$
\mathcal{I}(\mathbf{x}, t)=\alpha \frac{\sum_{i} \psi_{i} b_{i}(\mathbf{x}, t)+q f}{\sum_{i} \psi_{i} b_{i}(\mathbf{x}, t)+q}, \quad 0<f<1
$$

(b) the water-dependent biomass growth rate, $\mathcal{G}_{b_{i}}$ (see equation (2.7)), and (c) the overland water flow terms in the equation for $h$. According to equation (2.6) the infiltration rate increases monotonically with the biomass variables (since $f<1$ ), approaching the asymptotic value $\alpha$. As a 


\begin{tabular}{|cll|}
\hline Parameter & Units & Description \\
\hline \hline$K_{i}$ & $\mathrm{~kg} / \mathrm{m}^{2}$ & Maximum standing biomass \\
$Q$ & $\mathrm{~kg} / \mathrm{m}^{2}$ & $\begin{array}{l}\text { Biomass reference value beyond which infiltration rate } \\
\text { under a patch approaches its maximum }\end{array}$ \\
& & Biomass decay rate due to mortality and consumer pressure \\
$M_{i}$ & $\mathrm{yr}^{-1}$ & Infiltration rate in uniformly vegetated soil \\
$A$ & $\mathrm{yr}^{-1}$ & Soil water evaporation rate \\
$N$ & $\mathrm{yr}^{-1}$ & Root augmentation per unit biomass \\
$E_{i}$ & $\left(\mathrm{~kg} / \mathrm{m}^{2}\right)^{-1}$ & Biomass growth rate per unit soil water \\
$\Lambda_{i}$ & $\left(\mathrm{~kg} / \mathrm{m}^{2}\right)^{-1} \mathrm{yr}^{-1}$ & Soil water consumption rate per unit biomass \\
$\Gamma_{i}$ & $\left(\mathrm{~kg} / \mathrm{m}^{2}\right)^{-1} \mathrm{yr}^{-1}$ & Seed dispersal coefficient \\
$D_{B_{i}}$ & $\mathrm{~m}^{2} / \mathrm{yr}$ & Transport coefficient for soil water \\
$D_{W}$ & $\mathrm{~m}^{2} / \mathrm{yr}$ & Transport coefficient for soil water (inversely related to \\
$D_{H}$ & $\mathrm{~m}^{2} / \mathrm{yr}\left(\mathrm{kg}^{2} \mathrm{~m}^{2}\right)^{-1}$ & ground friction) \\
& & Lateral root length of a seedling \\
$S_{i}$ & $\mathrm{~m}$ & Topography function \\
$Z(\mathrm{X})$ & $\mathrm{mm}$ & Precipitation rate \\
$P$ & $\mathrm{~kg} / \mathrm{m}^{2} \mathrm{yr}^{-1}$ & Evaporation reduction due to shading \\
$R_{i}$ & - & Infiltration contrast between bare soil and vegetated soil \\
$f$ & - &
\end{tabular}

Table 1: A list of dimensional parameters, their units and their meaning. The subscript $i$ refers to traits of the $i$ th life form.

result, larger biomass implies higher soil-water content and therefore higher biomass-growth rates. The strength of this feedback can be controlled by the parameter $f$. Low values of $f$, modeling e.g. the existence of biogenic crusts [20], correspond to low infiltration rates in bare soil relative to the infiltration rates in vegetation patches. This infiltration contrast increases the surface water flow towards the vegetation patches, and thereby strengthens the infiltration feedback. The parameters $\psi_{i}$ quantify the relative contributions of the various species to the infiltration contrast.

\subsubsection{Root-augmentation feedback}

This is a positive feedback between the above-ground biomass and the below-ground roots. As the plant grows its root system extends in size and probes new soil regions where water can be taken up from. As a result the amount of water available to the plant increases and the plant grows even further. This feedback is captured by the nonlinear and nonlocal form of the biomass growth rate

$$
\begin{aligned}
\mathcal{G}_{b_{i}}(\mathbf{x}, t) & =\nu \lambda_{i} \int_{\Omega} g_{i}\left(\mathbf{x}, \mathbf{x}^{\prime}, t\right) \mathcal{F}\left[w\left(\mathbf{x}^{\prime}, t\right)\right] d \mathbf{x}^{\prime}, \\
g_{i}\left(\mathbf{x}, \mathbf{x}^{\prime}, t\right) & =\frac{1}{2 \pi \sigma_{i}^{2}} \exp \left[-\frac{\left|\mathbf{x}-\mathbf{x}^{\prime}\right|^{2}}{2\left[\sigma_{i}\left(1+\eta_{i} b_{i}(\mathbf{x}, t)\right)\right]^{2}}\right],
\end{aligned}
$$




\begin{tabular}{|cl||cl|}
\hline Quantity & Scaling & Quantity & Scaling \\
\hline \hline$b_{i}$ & $B_{i} / K_{i}$ & $p$ & $\Lambda_{1} P / N M_{1}$ \\
$w$ & $\Lambda_{1} W / N$ & $\gamma_{i}$ & $\Gamma_{i} K_{i} / M_{1}$ \\
$h$ & $\Lambda_{1} H / N$ & $\eta_{i}$ & $E_{i} K_{i}$ \\
$\nu$ & $N / M_{1}$ & $\rho_{i}$ & $R_{i}$ \\
$\lambda_{i}$ & $\Lambda_{i} / \Lambda_{1}$ & $\sigma_{i}$ & $S_{i} / S_{1}$ \\
$\mu_{i}$ & $M_{i} / M_{1}$ & $\delta_{b_{i}}$ & $D_{B_{i}} / M_{1} S_{1}^{2}$ \\
$\alpha$ & $A / M_{1}$ & $\delta_{w}$ & $D_{W} / M_{1} S_{1}^{2}$ \\
$q$ & $Q / K_{1}$ & $\delta_{h}$ & $D_{H} N / M_{1} \Lambda_{1} S_{1}^{2}$ \\
$\mathbf{x}$ & $\mathbf{X} / \mathbf{S}_{1}$ & $\psi_{i}$ & $Y_{i} K_{i} / K_{1}$ \\
$t$ & $M_{1} T$ & $\zeta$ & $\Lambda_{1} Z / N$ \\
\hline
\end{tabular}

Table 2: Relations between non-dimensional variables and parameters and their dimensional counterparts. Note that $\lambda_{1}=\mu_{1}=\sigma_{1}=1$.

where $\lambda_{i}$ represents the plant's growth rate per unit amount of soil water, the Gaussian kernel $g_{i}\left(\mathbf{x}, \mathbf{x}^{\prime}, t\right)$ represents the root system, and the integration is over the lateral roots domain $\Omega^{2}$ According to this form, the biomass growth rate depends not only on the amount of soil water at the plant location $\mathbf{x}$, but also on the amount of soil water in the neighborhood, $\mathbf{x}^{\prime}$, spanned by the plant's roots. Root augmentation in response to biomass growth is modeled by the biomassdependent width, $\left[\sigma_{i}\left(1+\eta_{i} b_{i}(\mathbf{x}, t)\right)\right]$, of the Gaussian function in equation (2.8) which provides a measure of the lateral root-system size. The parameter $\eta$ quantifies the root augmentation per unit biomass, beyond a minimal root-system size $\sigma_{i}$, which may represent the root size of a seedling. It measures the strength of root-augmentation feedback; the larger $\eta$ the stronger the feedback.

\subsubsection{Uptake feedback}

This is a negative feedback between biomass and water due to water-uptake by plants' roots. The depletion of soil-water at any given point is due to all plants whose roots extend to this point. The feedback is captured by the term $-\mathcal{G}_{w} w$ in the equation for $w$, where the water uptake rate, $\mathcal{G}_{w}$, increases with the biomass of all life forms according to

$$
\mathcal{G}_{w}(\mathbf{x}, t)=\sum_{i=1}^{n} \gamma_{i} \int_{\Omega} g_{i}\left(\mathbf{x}^{\prime}, \mathbf{x}, t\right) b_{i}\left(\mathbf{x}^{\prime}, t\right) d \mathbf{x}^{\prime} .
$$

Here, $\gamma_{i}$ is a parameter that measures the soil water uptake rate per unit biomass, associated with the $i$ th life form. Note that $g_{i}\left(\mathbf{x}^{\prime}, \mathbf{x}, t\right) \neq g_{i}\left(\mathbf{x}, \mathbf{x}^{\prime}, t\right)$.

\footnotetext{
${ }^{2}$ In practice we integrate over the entire domain.
} 


\subsubsection{Shading feedback}

This is a positive feedback between biomass and soil-water due to reduced evaporation at vegetation patches. The feedback is captured by the biomass dependence of the evaporation term, $-\mathcal{L} w$ in the equation for $w$, where

$$
\mathcal{L}(\mathbf{x}, t)=\frac{\nu}{\sum_{i=1}^{n}\left(1+\rho_{i} b_{i}\right)},
$$

$\nu$ is the soil-water evaporation rate in bare soil, and $\rho_{i}$ describes the reduction in the evaporation rate due to shading by the $i$ th life form. When the shading feedback is sufficiently weak we can use the linear approximation $\mathcal{L}(\mathbf{x}, t) \approx \nu \sum_{i=1}^{n}\left(1-\rho_{i} b_{i}\right)$.

\subsubsection{Pattern forming feedbacks}

The infiltration and the root-augmentation feedbacks both involve water transport. Thus, while accelerating the biomass growth in a vegetation patch these feedbacks inhibit the growth in the patch neighborhood, either by draining surface water through overland flow or by soil-water uptake through the spatially extended root system. This induces long range competition that can result in vegetation pattern formation. The shading feedback, on the other hand, does not involve water transport; the increase of soil-water content under a vegetation patch relative to its bare surrounding due to reduced evaporation does not involve the depletion of soil-water from the patch surroundings. As a result the shading feedback alone does not induce vegetation patterns.

\section{Basic landscape states along aridity gradients}

\subsection{Single life form}

We begin by considering a population of a single life form representing woody vegetation. We characterize the population by the biomass, $B_{1}(\mathbf{X}, T) d \mathbf{X}$, of all individuals in an infinitesimal area element $d \mathbf{X}$ around the point $\mathbf{X}=(X, Y)$ at time $T^{3}$. The corresponding non-dimensional biomass, $b_{1}=b(\mathbf{x}, t)$, satisfies Eqs. (2.1)-(2.3) with $n=1$. We assume that the growth time scale of the woody life form is typically long compared to time scales associated with seasonal variability. In the absence of significant long time-scale rainfall variability, e.g. droughts, we can take the precipitation rate $p$ to be a time-independent parameter that represents the mean annual rainfall rate. Since we consider drylands, $p$ and consequently $w$ are relatively small, justifying a linear approximation of the uptake function $\mathcal{F}(w) \approx w$. This may not be the case with other life forms, such as annuals, that can respond to fast rainfall variability.

\footnotetext{
${ }^{3}$ We do not specify any condition on the number of individuals that the biomass in any infinitesimal area represents. Quite often, even a single individual behaves in a continuous manner as far as its biomass is concerned. For example, a plant may respond to a precipitation down shift by letting only part of it die.
} 


\subsubsection{Plane topography}

For plane topography ( $\zeta=$ const.) the model equations (2.1)-(2.3) (with $n=1$ ) have two homogeneous stationary solutions representing bare soil and uniform vegetation. Their existence and linear stability ranges along a rainfall gradient are shown in the bifurcation diagram displayed in Fig. 2. The linear stability analysis leading to this diagram is described in [15].

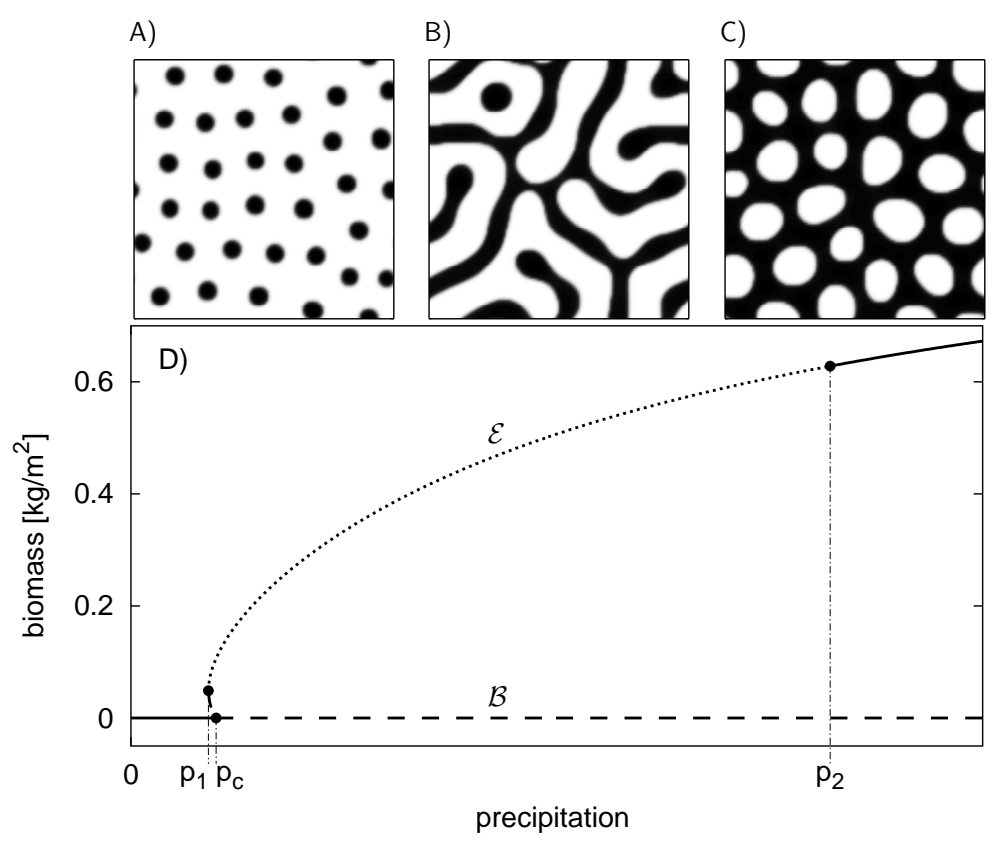

Figure 2: Vegetation states along a precipitation gradient. Frame D shows a bifurcation diagram of uniform states based on a linear stability analysis of the model equations ((2.1)-(2.3)) with $n=1$. The bare soil state $(\mathcal{B})$ is stable (solid line) at low precipitation and becomes unstable to uniform perturbations beyond $p_{c}$ (dashed line). The uniform vegetation state $(\mathcal{E})$ is stable at high precipitation and becomes unstable to non-uniform perturbations below $p_{2}$ (dotted line). Frames $\mathrm{A}, \mathrm{B}$, and $\mathrm{C}$ show typical patterns at increasing precipitation values in the range where uniform states are unstable: spots, stripes, and gaps (dark shades of gray represent high biomass). The patterns were obtained by numerical integration of the model equations ((2.1)-(2.3)) with $n=1$. The parameters used describe woody vegetation. From [30].

The bare soil solution, denoted in Fig. 2 by $\mathcal{B}$, is given by $b=0, w=p / \nu$ and $h=p / \alpha f^{4}$. It is linearly stable for $p<p_{c}=1$ and it loses stability at $p=1$ to uniform perturbations. The bifurcation is subcritical (supercritical) depending on whether the quantity $2 \eta \nu /[\nu(1-\rho)+\gamma]$ is greater (lower) than unity. The uniform vegetation solution, denoted by $\mathcal{E}$, exists for $p>1$ in the case of a supercritical bifurcation and for $p>p_{1}$ (where $p_{1}<1$ ) in the case of a subcritical bifurcation. It is stable, however, only beyond another threshold, $p=p_{2}>p_{1}$. As $p$ is decreased

\footnotetext{
${ }^{4}$ In accord with the view of $p$ as mean annual rainfall, also the value of $h$ in the bare soil solution should be interpreted as the mean annual value. See Ref. [17] for the consideration of intermittent rainfall.
} 
below $p_{2}$ the uniform vegetation solution loses stability to non-uniform perturbations in a finite wavenumber (Turing like) instability. These perturbations grow to form large amplitude patterns. The following sequence of basic patterns has been found at decreasing precipitation values for plane topography (see panels A-C in Figure 2): gaps, stripes and spots [3]. The spot and the gap patterns approach asymptotically hexagonal patterns. The stripe pattern generally takes the form of a labyrinthine pattern because of the system's isotropy in a plane topography.

Any pair of consecutive landscape states along the precipitation gradient has a range of bistability (coexistence of two stable states): bistability of bare soil with spots, spots with stripes, stripes with gaps, and gaps with uniform vegetation. The bistability of different landscape states has at least two important implications. First, it implies vulnerability to desertification, as environmental changes or disturbances may induce transitions to stable states of lower biological productivity $[2,3,31]$. Likewise, it implies the possible restoration of degraded areas by inducing a transition from a stable bare-soil state to a stable vegetation state. A common restoration practice on slopes is based on water harvesting by means of parallel contour ditches that accumulate runoff and along which vegetation is planted. The second implication of bistability is that it increases landscape diversity as patterns involving spatial mixtures of two distinct landscape states become feasible [29]. Of special interest is the bistability range of bare soil and spots where stable localized structures, consisting of a single or an isolated group of spots, exist [32, 33]. These states can be viewed as islands of the spot-pattern state in a bare-soil background, where the island boundaries are pinned in place in a range of precipitation values (rather than in a unique value representing the Maxwell point) [34]. The existence of such localized structures has been studied recently in simpler models, such as the Swift-Hohenberg and the complex Ginzburg-Landau equations. The pinning range is known as the "snaking region" because of the snake-like structure of the homoclinic orbits associated with the localized structures [35].

The patterns considered so far are not necessarily periodic or nearly periodic, as they may involve arbitrary spatial mixtures of different vegetation states, but they all contain a characteristic length - the size of a spot, the width of a stripe or the extent of a gap. There is a limit, however, where amorphous scale-free patterns are formed. This limit corresponds to conditions where water transport is infinitely faster than any process that absorb or exploit it. One realization of this limit is fast surface-water flow (large $D_{H}$ ) relative to the infiltration into vegetated soil (small $A$ ). Under this condition surface water can flow over long distances before infiltrating significantly into the soil, allowing for large vegetation patches whose size scales like $\sqrt{D_{H} P} / A$ [38]. This limit may not be attainable in practice but may be approached to the extent that it yields wide patch size distributions as observed in field studies [36, 37]. For more details about this limit and various realizations thereof the reader is referred to von Hardenberg et al. [38].

\subsubsection{Constant-slope topography}

The basic landscape states persist on constant slopes. There are, however, some differences [39] of which we mention two: (i) stripes reorientation themselves perpendicular to the slope direction to form parallel bands, and (ii) patterns migrate uphill. Both processes are demonstrated in Fig. 3 which shows the dynamics of an initially uniform biomass distribution. The slope introduces a pre- 
ferred direction, down-hill, that maximizes the interception of runoff at vegetation patches, thereby stabilizing vegetation bands that are oriented perpendicular to the slope. At relatively low precipitation rates low-wavenumber bands are formed. At higher precipitation rates higher wavenumbers appear but many of the lower wavenumber patterns remain stable. These states are likely to be related to the discrete families of Eckhaus stable solutions that have been found in simpler models of finite systems, such as the Ginzburg-Landau model [40]. Within the multistability range of band states, those states with higher wavenumbers are more biologically productive in the sense that more biomass is produced per unit of consumed soil-water. Higher wavenumber patterns, however, may be less resilient to environmental changes, such as prolonged droughts [39]. The migration uphill can be explained by the vegetation growth in the upper edge of the band due to accumulated runoff, and the vegetation die-out in the lower edge of the band due to loss of runoff downhill. Migrating bands on a slope have been found in earlier models as well, and also in field observations [12].

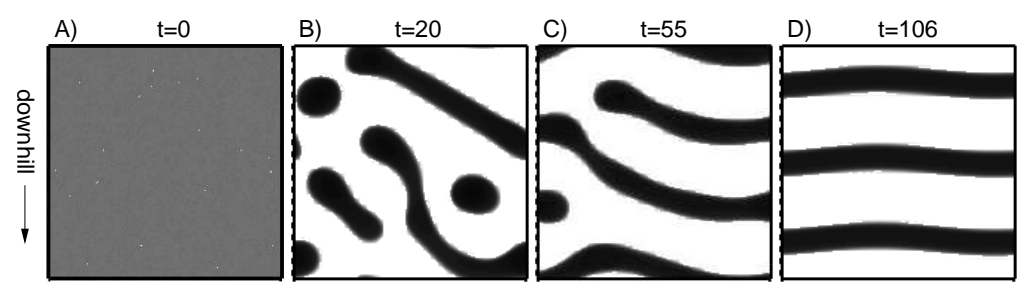

Figure 3: Development of vegetation bands traveling uphill from an unstable uniform vegetation state, obtained by numerical integration of the model equations (2.1)-(2.3) with $n=1$ on a constant slope. The different panels A-D shows snapshots of this process at different times ( $t$ in years). Dark shades of gray represent high biomass. The bands orient themselves perpendicular to the slope gradient and migrate uphill with typical speed of a few centimeters per year. From [15].

\subsection{Two life forms}

We now proceed to the level of a small community consisting of two life forms representing woody and herbaceous vegetation, with biomass variables $b_{1}$ and $b_{2}$, respectively. We distinguish between the two life forms by choosing the maximum standing biomass of the woody species to be an order of magnitude higher than that of the herbaceous species while its growth and mortality rates are taken to be significantly lower. We confine ourselves to the case of strong infiltration feedback $(f \ll 1)$ and moderate uptake feedback of the woody species $\left(\eta_{1} \sim \mathcal{O}(1)\right)$. These conditions are often realized in drylands where biological soil crusts increase the infiltration contrast and the woody vegetation consists of shrubs [22]. With this parameter choice we find that the herbaceous vegetation is strongly affected by the woody vegetation, but the woody vegetation is hardly affected by the herbaceous one. For simplicity, we still take the precipitation rate $p$ to be a constant parameter representing the mean annual rainfall. Although the herbaceous vegetation has biomass growth and decay rates that are fast enough to follow seasonal variability, the effect of the latter is quite often limited to the introduction of temporal biomass oscillations, with small shifts in threshold values. A detailed discussion of these effects is presented in Kletter et al. [17]. 


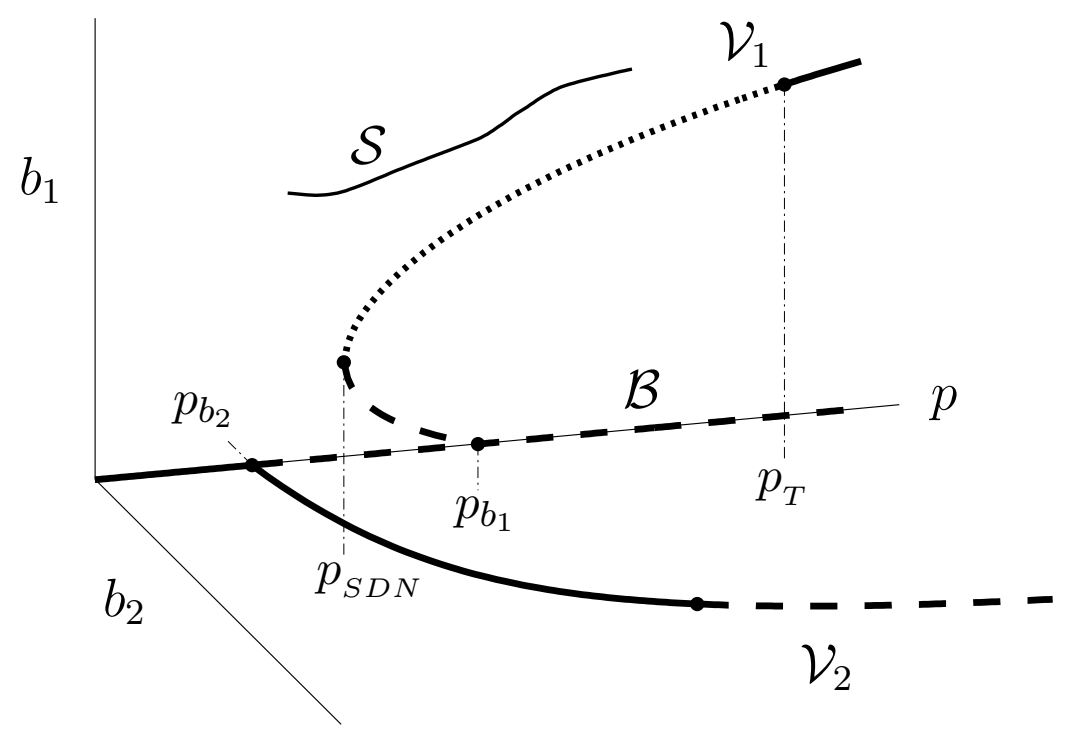

Figure 4: Bifurcation diagram showing homogeneous and pattern solutions of equations (2.1)(2.3) with $n=2$, representing a woody-herbaceous system. The solution branches $\mathcal{B}, \mathcal{V}_{1}$ and $\mathcal{V}_{2}$ represent, respectively, uniform bare-soil, uniform woody vegetation and uniform herbaceous vegetation. The branch $\mathcal{S}$ represents the amplitudes of spots patterns. Solid lines represent stable solutions, and dashed and dotted lines represent solutions unstable to uniform and non-uniform perturbations, respectively. The instability thresholds of the bare-soil solution, $p_{b_{1}}$ and $p_{b_{2}}$, are given by $p_{b_{1}}=1$ and $p_{b_{2}}=\mu_{2} / \lambda_{2}$. The points $p_{S D N}$ and $p_{T}$ denote, respectively, the saddle-node bifurcation and the finite-wavenumber instability along the uniform woody-vegetation branch $\mathcal{V}_{1}$. From [16].

Equations (2.1)-(2.3), for $n=2$ and plane topography, have four stationary uniform solutions:

- $\mathcal{B}$ : bare soil $\left(b_{1}=0, b_{2}=0\right)$.

- $\mathcal{V}_{1}$ : uniform woody vegetation $\left(b_{1} \neq 0, b_{2}=0\right)$.

- $\mathcal{V}_{2}$ : uniform herbaceous vegetation $\left(b_{1}=0, b_{2} \neq 0\right)$.

- $\mathcal{M}$ : uniform mixed woody-herbaceous vegetation $\left(b_{1} \neq 0, b_{2} \neq 0\right)$.

The first three solutions and their stability properties are displayed in the bifurcation diagram shown in Fig. 4 with $p$ as the control parameter. The uniform mixed-vegetation solution $\mathcal{M}$ is always unstable for the parameter regime of interest here and is not displayed. We refer the reader to Ref. [16] for more details about this analysis. Also shown is a non-uniform solution branch $(\mathcal{S})$ evaluated by numerically solving Eqs. (2.1)-(2.3). This solution branch describes a pure woody spot pattern at relatively low and high $p$ values, and a mixed woody-herbaceous spot pattern at intermediate $p$ values (see also section 5.). In the parameter regime considered here stable spot 
patterns coexist with stable uniform herbaceous vegetation, and possibly with the bare-soil solution as well. Additional non-uniform solutions, describing stripes and gap patterns, exist at higher precipitation values but are not shown in the bifurcation diagram.

If $\mu_{2} / \lambda_{2}<1$, the case shown in the bifurcation diagram, the bare-soil solution first loses stability to the growth of uniform herbaceous vegetation at $p=p_{b_{2}}=\mu_{2} / \lambda_{2}$. If $\mu_{2} / \lambda_{2}>1$, the bare-soil solution first loses stability to the growth of uniform woody vegetation $\left(\mathcal{V}_{1}\right)$ and the instability threshold is $p=p_{b_{1}}=1$. The uniform woody-vegetation solution, however, is unstable to non-uniform perturbations due to a Turing-like instability at $p=p_{T}$. The bare soil solution therefore destabilizes at $p=1$ to the spot-pattern solution $\mathcal{S}$.
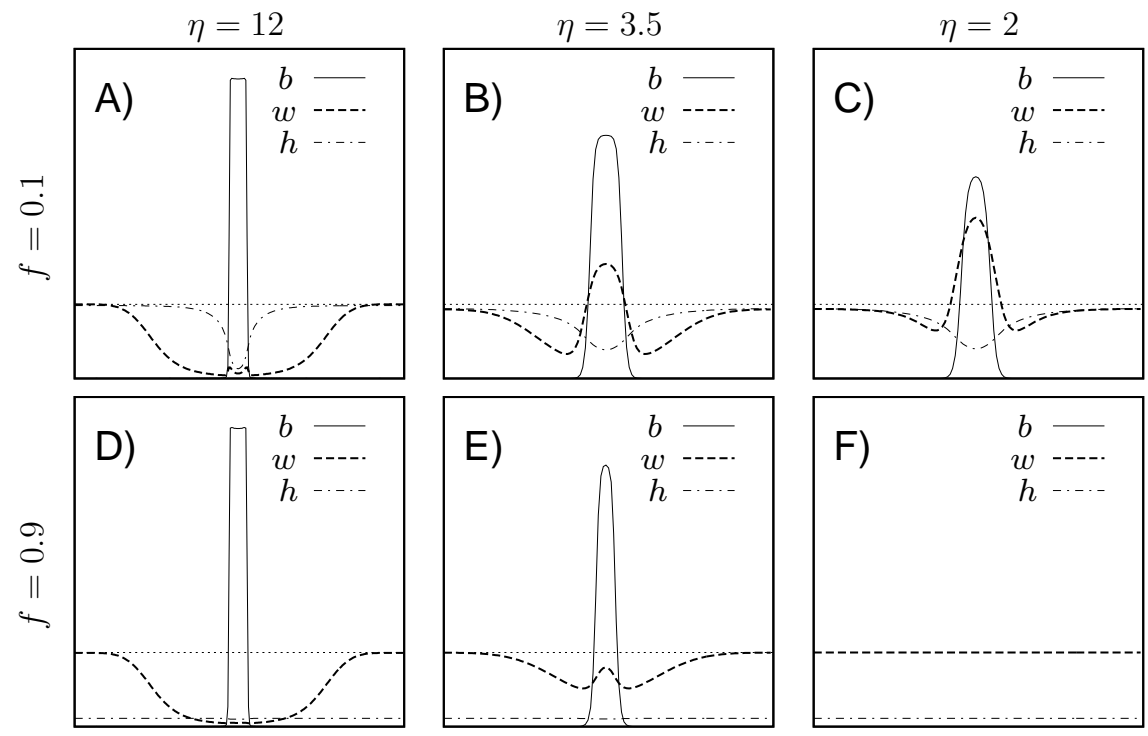

Figure 5: Spatial profiles of the variables $b, w$ and $h$ as affected by the parameters that control the infiltration feedback $(f)$ and the root-augmentation feedback $(\eta)$. The profiles are cross sections of two-dimensional simulations of the model equations (Eqs. (2.1)-(2.3) with $n=1$ ). In all panels, the horizontal dotted lines denote the soil-water level at bare soil. Strong infiltration feedback and weak root-augmentation feedback (panel C) leads to high soil-water concentration reflecting strong engineering. Strong root-augmentation feedback results in soil-water depletion and no engineering, irrespective of the infiltration-feedback strength (panels A,D). While the species characterized by $\eta=2$ is the best engineer under conditions of strong infiltration contrast (panel C), it leads to low system resilience; the engineer along with the micro-habitat it forms completely disappear when the infiltration contrast is strongly reduced, e.g. by crust removal (panel F). A species with somewhat stronger uptake feedback $(\eta=3.5)$ still acts as an ecosystem engineer (panel B) and also survives disturbances that reduce the infiltration contrast (panel E), thereby retaining the system's resilience. Panels A and D span a horizontal range larger than the range in all other panels. The vertical range in all panels is $[0,1] \mathrm{kg} / \mathrm{m}^{2}$ for the dimensional biomass variable, and $[0,187.5]$ $\mathrm{kg} / \mathrm{m}^{2}$ for the dimensional soil-water variable. From [15]. 


\section{Resource dynamics}

\subsection{Plants as ecosystem engineers}

Associated with the biomass patterns of a single life form are soil-water patterns. The spatial soilwater distribution is affected by the four feedbacks discussed in section 2.3.. The infiltration and shading feedbacks act to increase the soil-water content in the patch area (relative to bare soil) whereas the uptake and root-augmentation feedbacks act to deplete the soil-water content. The relative strengths of these feedbacks determine the actual soil-water distribution, and in particular, whether the soil-moisture in a patch is lower or higher than that in bare soil.

Figure 5 shows how the soil-water distribution is affected by varying the parameters that control the infiltration $(f)$ and the root-augmentation $(\eta)$ feedbacks in equations (2.1)-(2.3) with $n=1$. The other two feedbacks were kept unchanged. Shown are spatial profiles of $b$ and $w$ at decreasing values of $\eta$, and for two extreme values of $f: f \ll 1$ and $f \approx 1$. Decreasing values of $\eta$ represent species that grow shorter roots for the same growth of above ground biomass. Small $f$ values model high infiltration contrasts, that is, high infiltration rates in vegetation patches relative to bare soil, and may describe patchy landscapes with biological crusts covering the bare soil areas. Values of $f$ approaching unity model high infiltration rates everywhere as may be the case in an un-crusted sandy soil. As Panels B and C in Fig. 5 indicate, patches that concentrate the water resource, i.e. having soil-moisture higher than the moisture in bare soil, can be realized with high infiltration contrasts, $f=0.1$, and limited root extension capabilities, $\eta=3.5, \eta=2$.

The capability of a plant species to concentrate the water resource in its patch area has important consequences for the existence of other species, which may benefit from the ameliorated micro-environment that the patch forms. Indeed, woody patches in arid regions often facilitate the growth of herbaceous species, such as annuals [41, 42]. Species that have the capacity to modify the abiotic environment in ways that facilitate the growth of other species are called ecosystem engineers [43, 44]. Plant species capable of concentrating the limiting water resource can therefore be regarded as ecosystem engineers.

While a weak root-augmentation feedback enhances the engineering ability, it reduces the resilience of the ecosystem engineer (and all dependent species) to disturbances. Figure 5(F) shows the response of an engineer species with the highest engineering ability to concentrate water $(\eta=2$, Fig. $5(\mathrm{C})$ to a disturbance that strongly reduces the infiltration contrast $(f=0.9)$. In the following we refer to crust removal, but other disturbances that reduce the infiltration contrast will have similar effects. The engineer, and consequently the micro-habitat it forms, disappear altogether for two reasons: (i) surface water infiltrates equally well everywhere and the plant patch is no longer effective in trapping water, (ii) the engineer's roots are too short to collect water from the surrounding area. Resilient ecosystem engineers are obtained with strong infiltration feedbacks and moderate root-augmentation feedbacks $(\eta=3.5)$ as Fig. 5(E) shows. Removal of the crust (by increasing $f$ ) destroys the micro-habitats (soil-water content is smaller than the bare-soil's value) but the engineer persists. Once the crust recovers the ecosystem engineer resumes its capability to concentrate water and the micro-habitats recover as well. 

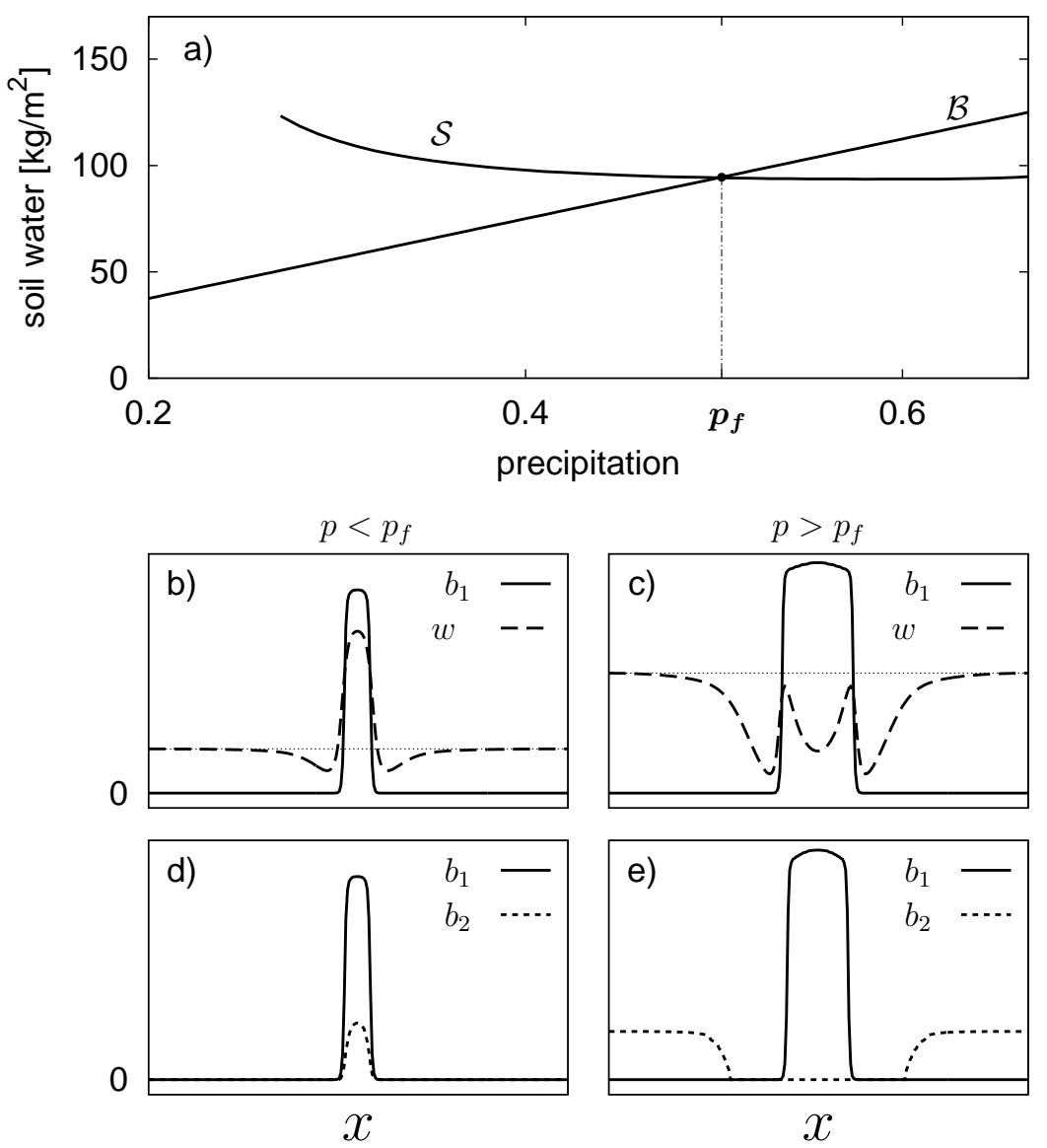

Figure 6: Solutions of the model equations for a single life form $(a, b, c)$ representing a woody engineer, and for two life forms $(\mathrm{d}, \mathrm{e})$, representing a woody-herbaceous system. The lines $\mathcal{B}$ and $\mathcal{S}$ in panel (a) show, respectively, the soil-water content in bare soil and the maximal content under a woody patch as functions of precipitation. The crossing point at $p=p_{f}$ represents a transition from negative engineering at high $p$ to positive engineering at low $p$. Panels $(\mathrm{b}, \mathrm{c})$ show spatial profiles of the engineer's biomass, $b_{1}$, and of the soil-water content, $w$, in the ranges of positive and negative engineering, respectively. Panels $(\mathrm{d}, \mathrm{e})$ show the response of an herbaceous life form, $b_{2}$, to the engineering of a woody life form, $b_{1}$. When $p>p_{f}$ (e) the herbaceous life form is excluded by the woody life form due to negative engineering, whereas for $p<p_{f}$ (d) the woody life form facilitates the growth of the herbaceous life form. From [15].

\subsection{Ecosystem engineering along a rainfall gradient}

The discussion in the previous section was limited to a single vegetation patch under fixed rainfall conditions. How does the engineering capacity of a given species change along a rainfall gradient? Figure 6a shows the dependence of the soil-water content on the precipitation rate for a species representing woody vegetation. The line $\mathcal{B}$ shows the soil-water content in bare soil while the line $\mathcal{S}$ shows the maximal water content under an engineer patch. The two lines intersect at $p=p_{f}$ 
suggesting a crossover from "negative" engineering at high precipitation $\left(p>p_{f}\right)$, where the soilwater content under a patch is lower than in bare soil, to "positive" engineering at low precipitation $\left(p<p_{f}\right)$, where the soil-water content under a patch exceeds that of bare soil. Figures $6(\mathrm{~b}, \mathrm{c})$ show examples of spatial profiles of biomass $\left(b_{1}\right)$ and soil-water $(w)$ in the negative-engineering range (c) and in the positive-engineering range (b). Note that the line $\mathcal{S}$ terminates at some low precipitation value. Below that value the ecosystem engineer $\left(b_{1}\right)$ no longer survives the dry conditions (localized spot solution of the woody life form no longer exists) and a transition (catastrophic shift) to bare soil occurs.

The model offers the following mechanism for this crossover. As the system becomes more arid, the engineer's patch area becomes smaller and the water uptake decreases significantly. The infiltration rate at the reduced patch area, however, decreases only slightly because of its weak biomass dependence for $b_{1} \gg q$ (see Eq. (2.6). As a result, a given area of the patch in a more arid environment traps nearly the same amount of surface water, but a significantly smaller amount of soil water is consumed in that area due to fewer individuals in the surrounding region and also due to the lower biomass. The outcome is an increased soil-water content at the patch area and stronger engineering. Two factors prevent the engineer from exhausting the soil water for its own growth: its maximum standing biomass, which limits the local growth, and the depletion of soil water in the immediate vicinity of the patch which prevents its expansion.

\subsection{Ecosystem engineering and spatial patterning}

So far we limited ourselves to the scale of a single patch. Are there processes at the landscape scale that affect ecosystem engineering? Figure 7 shows a pattern transition from spots to bands on a slope, in a precipitation range where the two patterns are stable. The transition is induced by a local biomass removal in the top most band. We note that the disturbance (biomass removal) should be strong enough to drive the system out of the attraction basin of the linearly stable banded pattern. The disturbance induces a chain process downhill that culminates in a transition to a spot pattern as panels B,C,D show. The biomass removal at the uppermost band allows for more runoff to accumulate at the band segment just below it. As a result this segment grows faster, draws more water from its surrounding and induces vegetation decay at the nearby band segments. The decay of the vegetation in the nearby segments allow for more runoff to accumulate at the next band downhill. The whole process continues repeatedly until the whole pattern transforms into a spot pattern. In the terminology of pattern formation theory, this chain process amounts to the invasion of a spot pattern into a stripe pattern through the propagation of a front that is biasymptotic to the two stable pattern states.

The lower panels in Figure 7 show the soil-water distributions along the transects denoted by the dashed lines in the upper panels. The transition to spots is accompanied by enhanced engineering; the soil-water content under a spot is higher than the content under a band. The mechanism of this behavior can be understood as follows. The spot pattern self-organizes to form a hexagonal pattern. As a result each spot experiences a bare area uphill which is twice as large as the bare area between successive bands, and therefore absorbs more runoff. In addition, the higher biomass density of spots (due to water uptake from all directions) increases the infiltration 


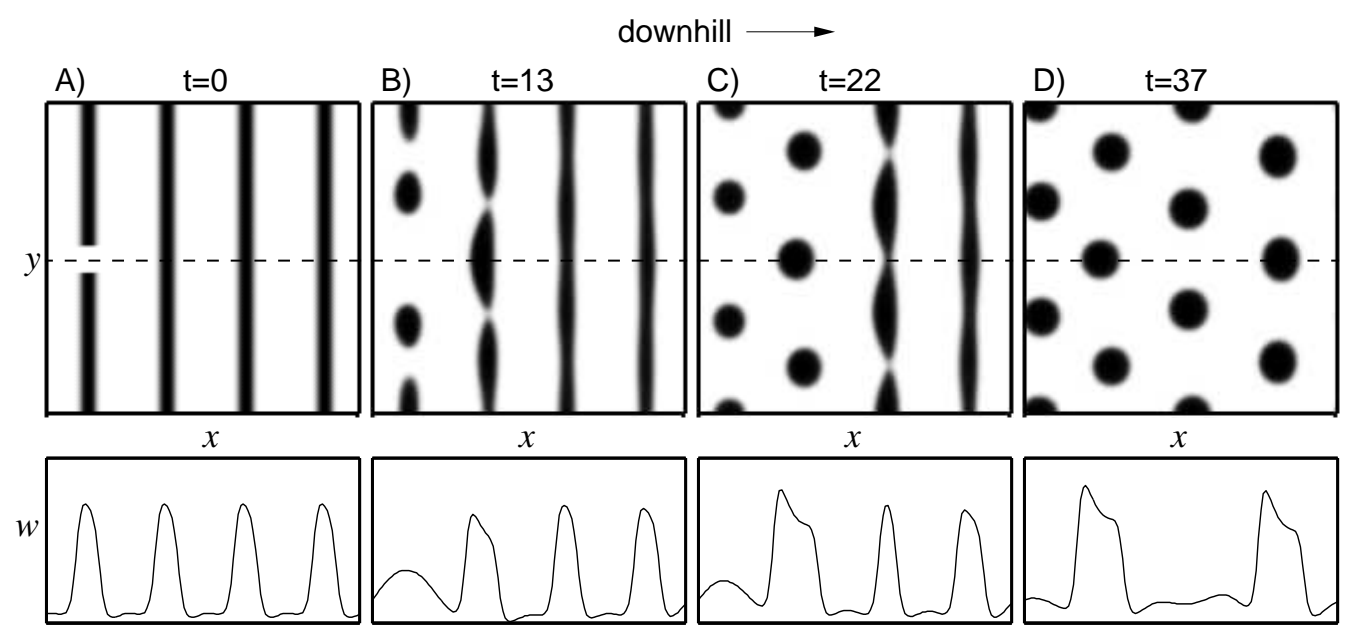

Figure 7: A pattern transition at the landscape level, from bands to spots, affects engineering at the single patch level. The transition is induced by a local clear-cutting disturbance (leftmost band in panel A), and is shown in panels A-D by displaying snapshots of biomass patterns (darker gray shades correspond to higher biomass density) at successive times ( $t$ in years). The patterns were obtained by solving numerically Eqs. (2.1)-(2.3) with $n=1$ at a fixed precipitation value where both band and spot patterns are stable. The initial biomass removal at the uppermost band induces a chain response culminating in an hexagonal spot pattern. The lower panels show soil water profiles along the transects denoted by the dashed lines in the corresponding upper panels. The soilwater content under spots is higher than the content under bands despite the fixed environmental conditions. A similar transition can be induced without a strong disturbance by a precipitation down shift to the monostability range of the spot pattern [30]. From [15].

contrast. Note that we kept here the precipitation rate fixed. The enhanced engineering is therefore a pure effect of pattern dynamics at the landscape level.

Engineering has also been found to be affected by the density of woody patches for fixed environmental conditions. In the bistability range of bare soil and spot patterns, where dense hexagonal packing of spots can be realized along with sparsely distributed spots, engineering could be restricted to the case of densely packed spots only. The mechanism of this behavior is similar to that of a single woody patch along a rainfall gradient, except that the water stress is induced by the strong competition among the densely packed spots.

\section{Response of herbaceous vegetation to woody engineers}

\subsection{Patch-scale response}

In section 4.1. we found the possible occurrence of a transition from negative to positive engineering along a rainfall gradient. In a woody-herbaceous system such a transition may imply a transition from competitive inter-specific plant interactions to facilitative interactions. Figure 
6(d,e) indeed demonstrates such a transition with appropriate realizations of an herbaceous life form. At high precipitation rates the herbaceous life form is excluded from the woody patch area and its neighborhood, whereas at low precipitation rates the only place where it can survive the dry conditions is the micro-habitat formed by the woody patches. Obviously, other realizations of the herbaceous life form in the model equations can give rise to situations where herbs are not excluded by woody plants at high precipitation rates, or can grow also in the open areas (away from woody patches) at low precipitation rates. Thus, in a system consisting of a community of herbs, the transition from competition to facilitation is likely to involve higher herbaceous biomass in the open areas when the precipitation rate is high, and higher herbaceous biomass under storey (i.e. in woody patches), when the precipitation rate is low. This prediction is in line with recent field observations along rainfall gradients and along other gradients of environmental stress $[45,41,46,47,42]$.

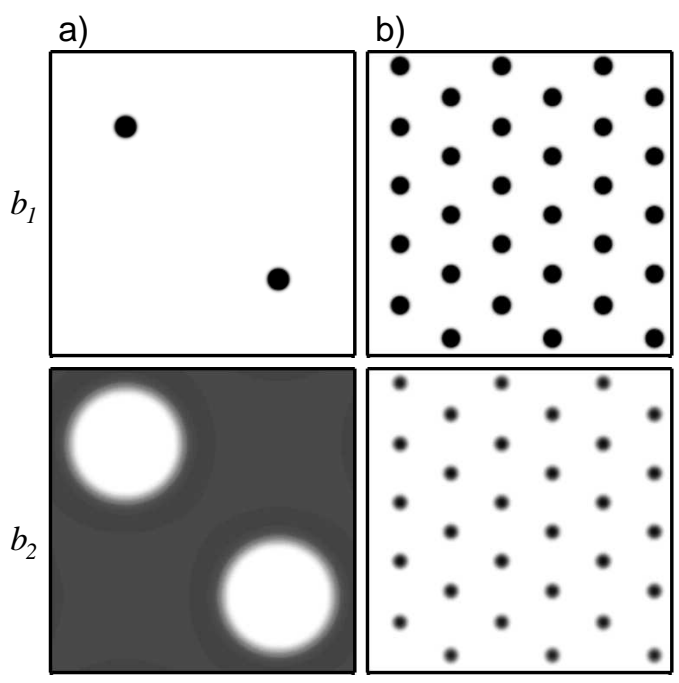

Figure 8: Solutions of the model equations for a woody-herbaceous systems showing a change from competition to facilitation associated with diluting woody-patch patterns. Shown are the spatial distributions of the woody and herbaceous biomass variables, $b_{1}$ and $b_{2}$, for sparsely scattered woody patches (a) and for a densely packed woody-patch pattern (b). For the chosen environment and species traits, the herbaceous life form is excluded from the woody patches and their close neighborhoods when the patches are sparsely scattered, but grows under storey when the patches are closely packed. From [16].

\subsection{Landscape-scale response}

At the landscape level changes from competition to facilitation can be induced solely by spatial patch organization (even for fixed environmental conditions). As discussed in section 3.2., the model equations have a bistability precipitation range of uniform herbaceous vegetation (with no 
woody vegetation) and a woody spot pattern. In this range a variety of spatially mixed patterns involving the two vegetation states exist. In particular, states with sparsely distributed woody patches can be realized along with states of densely packed woody patches. This change in spatial woody-patch organization can affect the growth areas of herbaceous vegetation, as Fig. 8 shows. While in a sparse patch pattern herbaceous vegetation is excluded by the woody life form and grow only in the open areas (Fig. 8(a)), in dense patch patterns the woody life form acts as an ecosystem engineer and facilitates the growth of the herbaceous life form under storey (Fig. 8(b)). In section

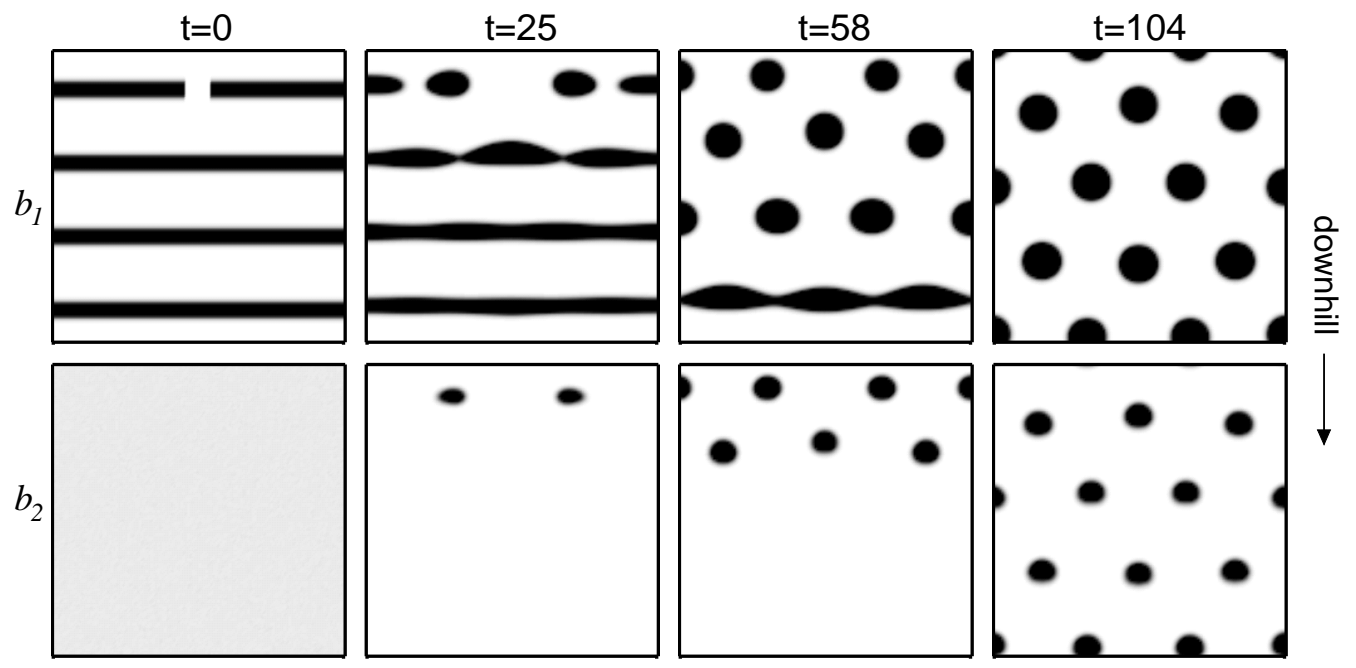

Figure 9: Facilitation induced by a pattern transition at the landscape level. Shown is a sequence of snapshots at different times ( $t$ is in years) obtained by solving Eqs. (2.1)-(2.3) (with $b_{2}$ small and randomly distributed at $t=0$ ). The snapshots describe a transition from vegetation bands to vegetation spots on a slope induced by a local clear cut along one of the bands. In the banded pattern the woody life form $\left(b_{1}\right)$ excludes the herbaceous life form $\left(b_{2}\right)$, but in the spotted pattern they coexist due to enhanced runoff concentration in woody spots. From [16].

4.3. we found that global pattern transitions from bands to spots on a slope can enhance local engineering at the patch scale. This enhanced engineering can facilitate the growth of herbaceous vegetation in woody patches as Fig. 9 demonstrates. Shown in the figure is the same transition as in Fig. 7 with the additional response of the herbaceous life form. The transition involves, in effect, a facilitation front propagating downhill; as bands gradually break into spots, patches with higher soil-water content are formed, facilitating the growth of herbaceous vegetation.

\section{Conclusion}

In this review I described the progress we have made in developing a platform of spatially explicit nonlinear models for studying the dynamics of water limited landscapes. The platform has first been used to study self-organized vegetation patchiness [4]. Five basic vegetation states have 
been identified along the rainfall gradient [3, 14]: uniform vegetation, gaps in uniform vegetation, stripes, vegetation spots, and bare soil. The gap and the spot patterns approach asymptotically hexagonal forms. In isotropic plane topography the stripes generally form labyrinthine patterns, but on slopes they reorient perpendicular to the slope direction and migrate uphill. Another outcome of the model is the existence of bistability precipitation ranges between any consecutive pair of vegetation states: uniform vegetation and gaps, gaps and stripes, stripes and spots, and spots and bare soil. These bistability ranges give rise to a wider variety of patterns involving spatial mixtures of different vegetation states.

Linear stability analysis of the uniform vegetation state shows that the destabilization of this state to gap patterns (as the precipitation rate is decreased) involves a stationary finite-wavenumber instability. The other uniform state, bare soil, loses stability (as the precipitation rate is increased) in a stationary uniform instability. The detailed bifurcation structure of the series of instabilities from uniform vegetation to bare soil has not been evaluated yet, mainly because of the difficulty to apply iterative methods to the model. It is very likely, however, that snaking regions $[35,33]$ exist in the bistability ranges of bare soil and spot patterns, and of uniform vegetation and gap patterns, that account for the existence of stable isolated vegetation spots in bare soil (or localized groups of them), and of isolated gaps in uniform vegetation.

All the patterns described above have characteristic length scales associated with the long (but finite) competition range induced by the infiltration and root-augmentation feedbacks (see section 2.3.5.). Patterns with no characteristic scales $[37,36]$ are obtainable in the model under conditions of global competition. Such conditions can be realized when the time scale associated with water transport is much shorter than the time scales associated with water exploitation or absorption [38].

Associated with biomass patterns are spatial soil-water distributions. These distributions are strongly affected by the engineering capacity of the woody life form. Ecosystem engineering can result from patch-scale processes and landscape-scale processes. At the patch scale engineering develops when the infiltration contrast is strong $(f \ll 1)$ and the root-augmentation is weak $(\eta \sim$ $\mathcal{O}(1)$ ). Engineering also develops upon increasing aridity (for fixed $f$ and $\eta$ ). At the landscape scale engineering depends on the woody-patch density; while sparsely packed patches deplete the soil-water content, densely packed patches can concentrate it. Engineering also depends on the type of the woody pattern; a transition from bands to spots on a slope can enhance engineering.

These ecosystem engineering effects were found to have direct implications on the colonization and growth of herbaceous vegetation. Studies of single-patch dynamics, using the two-life-form model, indicate that inter-specific woody-herbaceous plant interactions can change from competitive to facilitative along a rainfall gradient, due to the development of positive engineering at low precipitation rates. At the landscape level, colonization and growth of the herbaceous life form could be induced by increasing patch density or by inducing a pattern transition from bands to spots on a slope.

The model platform captures several aspects of ecosystem complexity, including processes occurring across different levels of organization and spatial scales. It captures bottom-up processes, such as self-organized patchiness, where plant interactions at the patch scale give rise to spatial patterns at the landscape scale. It also captures top-down processes, where pattern transitions at the landscape scale affect inter-specific interactions at the patch scale. More significantly, it high- 
lights the complex nature of ecological processes and suggests directions for studying them. For example, studies of the effects of climate changes or disturbances on species diversity, should not be restricted to the organism level and to the scale of a single patch. There are indirect processes, involving higher levels of organization and spatial scales, that bear on this question. As demonstrated in Figs. 7 and 9, woody pattern transitions at the landscape scale can change the soil-water content at the patch scale in ways that affects the colonization and growth of herbaceous vegetation.

Addressing questions of this type calls for methods of deriving community level properties such as species diversity or diversity-resource relations. The model equations (2.1)-(2.3), for a large number of life forms $n$, provide the general basis, but what plant traits should be used to distinguish among the life forms, and can their number be reduced for given environmental contexts, are questions that need further consideration. Further developments of the platform itself are needed too, including integration with other model studies. Equations (2.1)-(2.3) are two-dimensional in space. Inclusion of a soil-depth dimension is significant for several contexts including ecosystem engineering associated with hydraulic lift [48], and species coexistence due to water uptake from soil layers of different depths. Extending equations (2.1)-(2.3) to include erosion-deposition processes [19] is another direction for model development, especially significant in the study of desertification.

Finally, a few notes about solving the model equations numerically. We mostly solved the equations for flat and constant-slope topographies. Two bottlenecks slow down the integration time: the separation of time scales, with $h$ varying on a time scale much shorter than $w$ and $b$, and the root-kernel terms which cannot be rewritten as convolutions because of their biomass dependence. The time-scale problem can be resolved by setting $\partial h / \partial t=0$ and solving the equation iteratively [49]. The root-kernel problem can be resolved by approximating each kernel as a linear superposition of instances of the same kernel evaluated at constant biomass values for which the convolution theorem holds. Optimal values for the coefficients in the superposition can be derived by the minimization of an appropriately defined error $[49,50]$. Another concern is related to the $h$ equation for variable topographies, which has to be solved using appropriate numerical approaches to avoid numerical instabilities [27].

\section{Acknowledgement}

This review describes collaborations with students and colleagues, including in particular Erez Gilad, Jost von Hardenberg, Assaf Kletter, Jonathan Nathan, Antonello Provenzale, Moshe Shachak and Hezi Yizhaq. The author wish to thank Mauricio Santillana for helpful discussions. The research has been supported by the James S. McDonnell Foundation, and the Israel Ministry of Science and Technology.

\section{References}

[1] M. Shachak, B. Boeken, E. Groner, R. Kadmon, Y. Lubin, E. Meron, E.G. Neeman, A. Perevolotsky, Y. Shkedy, E. Ungar. Woody species as landscape modulators and their effect 
on biodiversity patterns. BioScience, 58 (2008), 209-221.

[2] M. Scheffer, S. Carpenter, J.A. Foley, C. Folke, B. Walkerk. Catastrophic shifts in ecosystems. Nature, 413 (2001), 591-596.

[3] J. von Hardenberg, E. Meron, M. Shachak, Y. Zarmi. Diversity of Vegetation Patterns and Desertification. Phys. Rev. Lett., 87 (2001), 198101.

[4] M. Rietkerk, S.C. Dekker, P.C. de Ruiter, J. van de Koppel. Self-organized patchiness and catastrophic shifts in ecosystems. Science, 305 (2004), 1926-1029.

[5] M. Loreau, S. Naeem, P. Inchausti, J. Bengtsson, J.P. Grime, A. Hector, D.U. Hooper, M.A. Huston, D. Raffaelli, B. Schmid, D. Tilman, D.A. Wardle. Biodiversity and Ecosystem Functioning: Current Knowledge and Future Challenges. Science, 294 (2001), 804-808.

[6] Millennium Ecosystem Assessment. Ecosystems and Human Well-being: Desertification Synthesis. World Resources Institute, Washington, DC. 2005.

[7] Millennium Ecosystem Assessment. Ecosystems and Human Well-being: Biodiversity Synthesis. World Resources Institute, Washington, DC. 2005.

[8] N. Barbier, P.Coutron, J. Lejoly, V. Deblauwe, O. Lejeune. Self-organized vegetation patterning as a fingerprint of climate and human impact on semi-arid ecosystems. Journal of Ecology, 94 (2006), 537-547.

[9] N. Barbier, P. Coutron, R. Lefever, V. Deblauwe, O. Lejune. Spatial decoupling of facilitation and competition at the origin of gapped vegetation patterns. Ecology, 89 (2008), 1521-1531.

[10] I. Stavi, H. Lavee, E.D. Ungar, P. Sarah. Ecogeomorphic feedbacks in semiarid rangelands: A review. Pedosphere, 19 (2009) 217-229.

[11] See the following review and references therein: F. Borgogno, P. D'Odorico, F. Laio, L. Ridolfi. Mathematical models of vegetation pattern formation in ecohydrology. Reviews of Geophysics, 47 (2009), RG1005.

[12] C. Valentin, J.M. d'Herbès, J. Poesen. Soil and water components of banded vegetation patterns. Catena, 37 (1999), 1-24.

[13] V. Deblauwe, N. Barbier, P. Couteron, O. Lejeune, J. Bogaert. The global biogeography of semi-arid periodic vegetation patterns. Global Ecol. Biogeogr., 17 (2008), 715-723.

[14] E. Gilad, J. von Hardenberg, A. Provenzale, M. Shachak, E. Meron. Ecosystem Engineers: From Pattern Formation to Habitat Creation. Phys. Rev. Lett., 93 (2004), 0981051.

[15] E. Gilad, J. von Hardenberg, A. Provenzale, M. Shachak, E. Meron. A mathematical Model for Plants as Ecosystem Engineers. J. Theor. Biol., 244 (2007), 680-691. 
[16] E. Gilad, M. Shachak, E. Meron. Dynamics and spatial organization of plant communities in water limited systems. Theor. Pop. Biol., 72 (2007), 214-230.

[17] A.Y. Kletter, J. von Hardenberg, E. Meron, A. Provenzale. Patterned vegetation and rainfall intermittency. J. Theor. Biol., 256 (2009), 574-583.

[18] R. Matyssek, H. Schnyder, E.F. Elstner, J.C. Munch, H. Pretzsch, H. Sandermann. Growth and parasite defence in plants: the balance between resource sequestration and retention: in lieu of a guest editorial. Plant Biol., 4 (2002), 133-136.

[19] P.M. Saco, G.R. Willgoose, G.R. Hancock. Eco-geomorphology of banded vegetation patterns in arid and semi-arid regions. Earth Syst. Sci.Hydrol., 11 (2007), 1717-1730.

[20] S.E. Campbell, J.S. Seeler, S. Glolubic. Desert crust formation and soil stabilization. Arid Soil Res. and Rehab. 3 (1989), 217-228.

[21] N.E. West. Structure and function in microphytic soil crusts in wildland ecosystems of arid and semi-arid regions. Adv. Ecol. Res., 20 (1990), 179-223.

[22] D.J. Eldridge, E. Zaady, M. Shachak. Infiltration through three contrasting biological soil crusts in patterned landscapes in the Negev, Israel. Catena, 40 (2000), 323-336.

[23] S.C. Dekker, M. Rietkerk, M.F.P. Bierkens. Coupling microscale vegetation-soil water and macroscale vegetation-precipitation feedbacks in semiarid ecosystems. Global change biology, 34 (2007), 671-678.

[24] S. Thompson, G. Katul, S. M. McMahon. Role of biomass spread in vegetation pattern formation within arid ecosystems. Water Resour. Res., 44 (2008), W10421.

[25] R. Nathan, R. Casagrandi. A simple mechanistic model of seed dispersal, predation and plant establishment: Janzen-Connell and beyond. Journal of Ecology, 92 (2004), 733-746.

[26] C.S. Holling. The functional response of invertebrate predators to prey density. Mem. Entomol. Soc. Canada, 48 (1966), 1-86.

[27] M. Santillana, C. Dawson. A numerical approach to study the properties of solutions of the diffusive wave approximation of the shallow water equations. Computational Geosciences, 14 (2010), 31-53.

[28] J.L. Vázquez. The porous medium equation. Mathematical theory. Oxford University Press, Oxford, 2006.

[29] E. Meron, E. Gilad, J. von Hardenberg, M. Shachak, Y. Zarmi. Vegetation Patterns Along a Rainfall Gradient. Chaos, Solitons and Fractals, 19 (2004), 367-376.

[30] E. Meron, E. Gilad. Dynamics of plant communities in drylands: A pattern formation approach. In Complex Population Dynamics: Nonlinear Modeling in Ecology, Epidemiology and Genetics. Eds: B. Blasius, J. Kurths, and L. Stone, p. 49-76, World-Scientific, 2007. 
[31] M. Rietkerk, F. van den Bosch, J. van de Koppel. Site-specific properties and irreversible vegetation changes in semi-arid grazing systems. Oikos, 80 (1997), 241-252.

[32] E. Meron, H. Yizhaq, E. Gilad. Localized structures in dryland vegetation: forms and functions. Chaos, 17 (2007), 037109.

[33] M. Tlidi, R. Lefever, A. Vladimirov. Vegetation Clustering, Localized Bare Soil Spots and Fairy Circles. In Dissipative solitons: from optics to biology and medicine. Lecture Notes in Physics, vol. 751 Springer, 2008.

[34] Y. Pomeau. Front motion, metastability and subcritical bifurcations in hydrodynamics. Physica D, 23 (1986), 3-11.

[35] E. Knobloch. Spatially localized structures in dissipative systems: open problems. Nonlinearity, 21 (2008), T45-T60.

[36] S. Kéfi, M. Rietkerk, C.L. Alados, Y. Pueyo, V.P. Papanastasis, A. ElAich, P.C. de Ruiter. Spatial vegetation patterns and imminent desertification in Mediterranean arid ecosystems. Nature, 449 (2007), 213-216.

[37] T.M. Scanlon, K.C. Kelly, S.A. Levin, I. Rodriguez-Iturbe. Positive feedbacks promote power-law clustering of Kalahari vegetation. Nature, 449 (2007), 209-212.

[38] J. von Hardenberg, A.Y. Kletter, H. Yizhaq, J. Nathan, E. Meron. Periodic vs. scale-free patterns in dryland vegetation. Proc. R. Soc. B, 277 (2010), 1771-1776.

[39] H. Yizhaq, E. Gilad, E. Meron. Banded vegetation: Biological Productivity and Resilience. Physica A, 356 (2005), 139-144.

[40] L.S. Tuckerman, D. Barkley. Bifurcation analysis of the Eckhaus instability. Physica D, 46 (1990), 57-86.

[41] F.I. Pugnaire, M.T. Luque. Changes in plant interactions along a gradient of environmental stress. Oikos, 93 (2001), 42-49.

[42] C. Holzapfel, K. Tielborger, H.A. Parag, J. Kigel, M. Sternberg. Annual plant-shrub interactions along an aridity gradient. Basic Appl. Ecol., 7 (2006), 268-279.

[43] C.G. Jones, J.H Lawton, M. Shachak, Organisms as ecosystem engineers, Oikos, 69 (1994), 373-386.

[44] C.G. Jones, J.H Lawton, M. Shachak. Positive and negative effects of organisms as ecosystem engineers. Ecology, 78 (1997), 1946-1957.

[45] R.M. Callaway, L.R. Walker. Competition and facilitation: a synthetic approach to interactions in plant communities. Ecology, 78 (1997), 1958-1965. 
[46] F.T. Maestre, S. Bautista, J. Cortina. Positive, negative and net effects in grassshrub interactions in Mediterranean semiarid grasslands. Ecology, 84 (2003), 3186-3197.

[47] C.M. Crain, M.D. Bertness M.D.. Ecosystem Engineering across Environmental Gradients: Implications for Conservation and Management. Bioscience, 56 (2006), 211-218.

[48] See for example F. Ludwig, T.E. Dawson, H. Kroon, F. Berendse, H.H.T. Prins. Hydraulic lift in Acacia tortilis trees on an East African savanna. Oecologia, 134 (2003), 293-300.

[49] E. Gilad. Mathematical models for vegetation patterns and biodiversity. $\mathrm{Ph} . \mathrm{D}$. thesis, BenGurion University (2006).

[50] E. Gilad, J. von-Hardenberg. A fast algorithm for convolution integrals with space and time variant kernels. Journal of Computational Physics, 216 (2006) 326-336. 